\section{Duke University Medical Center, Durham, North Carolina 27710, USA Department of Medicine R J Folz C A Piantadosi}

Department of Radiology

E K Folz

Correspondence to Rodney Folz, MD, PhD, Box 2620/Room 339, Medical Science Research Building, Durham, NC 27710, USA

Accepted 23 July 1997

\section{Spontaneous intercostal bulge}

\author{
Rodney J Folz, Claude A Piantadosi, Emily K Folz
}

A 48-year-old man had been diagnosed with severe chronic obstructive pulmonary disease and referred to our pulmonary clinic. His medical history revealed a $60-80$ pack year smoking history and long-standing heavy alcohol consumption. He worked as an upholsterer, with exposure to glues and paints for 20 years. Tuberculin skin testing was negative with a positive Candida control. Sputum Gram-stain revealed many polymorphonuclear leukocytes and was AFB-stain positive. Culture growth indicated mycobacterium other than tuberculosis identified as Runyoun's Group III. Drug sensitivity testing demonstrated $0 \%$ resistance to the combination of isoniazid, rifampin, and ethionamide.

His clinical condition gradually deteriorated despite antimicrobial therapy. Pulmonary function testing in 1994 demonstrated severe obstructive lung disease with a $\mathrm{D}_{\mathrm{L}} \mathrm{CO}$ of $29 \%$ predicted $(8.5$ $\mathrm{ml} / \mathrm{min} / \mathrm{mmHg}$ ). One month later, he developed acute bronchitis and during a severe coughing spell he noticed the acute onset of bulging of his left anterior chest wall. The bulge rapidly expanded during manoeuvres which increased intrathoracic pressure and rapidly reduced with cessation of these manoeuvres. In addition to the expansion and contraction of this chest wall bulge, physical examination was remarkable for sonorous breath sounds and the sound of 'gurgling' fluid within the bulge. A chest X-ray is shown in figure 1. A chest computed tomography (CT) scan (figure 2) was obtained in the semi-prone position during a Valsalva manoeuvre.

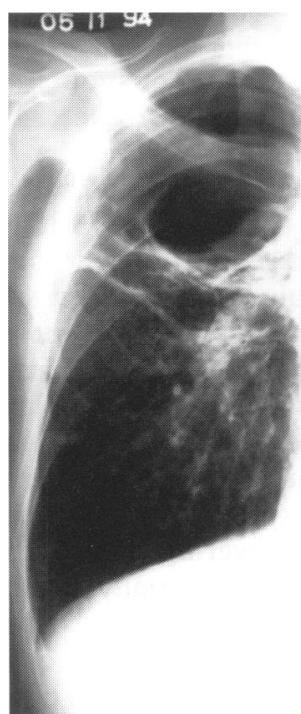

Figure 1 Chest X-ray
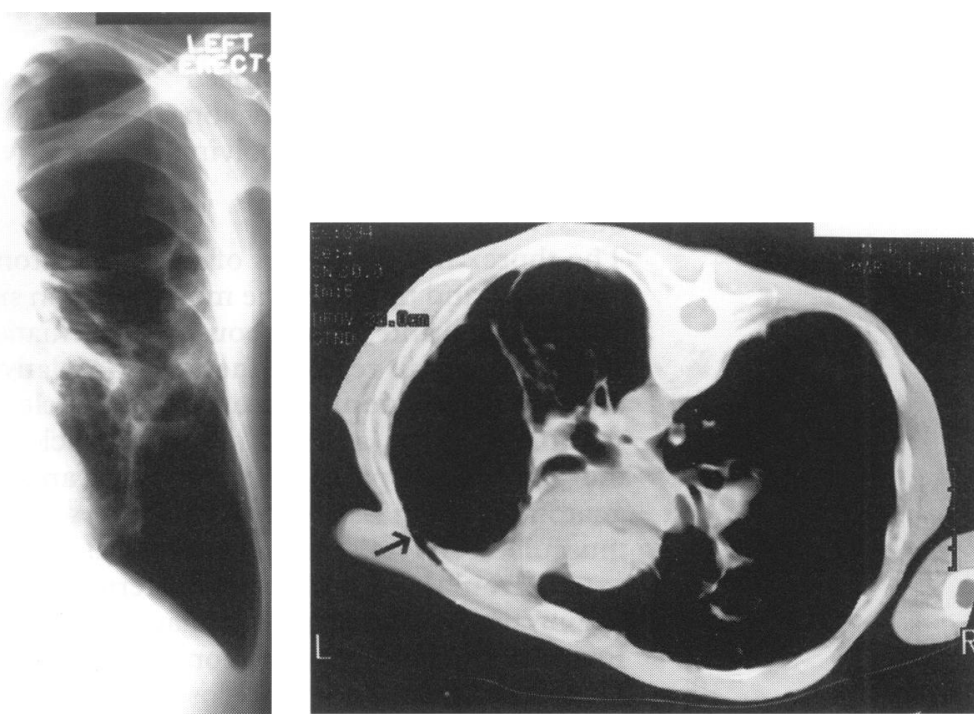

Figure 2 Chest CT scan in the semi-prone position during a Valsalva manoeuvre

\section{Questions}

1 Identify the pertinent findings in the chest X-ray (figure 1) and CT scan (figure 2).

2 What is the clinical diagnosis?

3 How might one classify these lesions?

4 List three sites of relative anatomic weakness in the chest wall. 


\begin{abstract}
Answers
QUESTION 1

The chest X-ray demonstrated hyperinflation, severe bullous emphysema, and cavities containing air-fluid levels. The chest CT scan in the semi-prone position during a Valsalva manoeuvre demonstrates severe bullous change in the left lung and a small cavity in the right lung. The arrow indicates herniation of a large bulla through the left anterior second and third rib interspace.
\end{abstract}

QUESTION 2

The diagnosis is spontaneous intercostal herniation of a lung bulla.

QUESTION 3

The original classification of extrathoracic lung herniation by Morel-Lavallee in 1849 is comprehensive and often still cited in the literature. The classification describes lung herniation according to location - cervical, thoracic, or diaphragmatic - and according to aetiology - congenital or acquired. Acquired lung hernias are further divided into traumatic, consecutive, spontaneous, and pathological. Only one case of diaphragmatic lung herniation has been described. ${ }^{1}$

The term consecutive hernia is confusing; it was used to define those hernias developing some time after a focal chest wall injury. These cases should be categorised as traumatic. Pathological hernias develop secondary to neoplasm or infection involving the chest wall.

\section{QUESTION 4}

The thorax has three sites of relative anatomic weakness, and these are the most common sites of congenital and spontaneous lung herniation. In the cervical region, there is a relatively unsupported region between the sternocleidomastoid and the anterior scalene muscles. In the intercostal spaces, there are two areas of anatomic weakness created by the absence of musculature. The external intercostal muscles extend from the rib tubercles posteriorly to the beginnings of the costal cartilages anteriorly. From there, they continue along the costal cartilage as the anterior intercostal membranes. The internal intercostal muscles extend from the sternum to the angles of the ribs, from which they continue as the posterior intercostal membranes to the vertebral column. It is at these sites created by the absence of the external intercostal muscles anteriorly, and the absence of the internal intercostal muscles posteriorly, that most spontaneous thoracic lung herniations occur.

\section{Discussion}

Approximately 264 cases of lung herniation have been reported in the literature. ${ }^{23}$ Of these, an estimated $52 \%$ were post-traumatic, $18 \%$ were congenital, and $29 \%$ were spontaneous. ${ }^{24}$ Of 11 spontaneous hernias reported by Hiscoe and Digman, eight were cervical and three thoracic. ${ }^{4}$ They contrasted their findings to those of Urbach in which seven of 25

\section{Classification of lung hernias}

According to location

- cervical

- thoracic

- diaphragmatic

According to aetiology

- congenital

- acquired: traumatic, consecutive, spontaneous, pathological

spontaneous hernias were cervical and 18 thoracic. ${ }^{4}$ These reports suggest that approximately half of the cases of spontaneous lung herniations are intercostal.

Spontaneous hernias have resulted from a variety of conditions associated with an excessive increase in intrathoracic pressure. These conditions include chronic bronchitis and emphysema, pertussus, straining during labour, heavy lifting, and blowing glass or musical instruments. ${ }^{56}$ The mechanism of spontaneous lung herniation in chronic bronchitis and emphysema is thought to be related to lung hyperinflation, refractory cough, and possibly chronic steroid use leading to muscle weakness. ${ }^{27}$ Spontaneous hernias have also been described in patients with congenital absence of chest wall musculature. ${ }^{6}$ Only one other case of spontaneous herniation of a lung bulla has been described. This occurred in the cervical region of a 60 -year-old woman with bullous emphysema. ${ }^{8}$

Spontaneous lung herniation usually presents clinically as the acute onset of a focal bulge associated with pain. Less commonly, a patient may present with progressive painless enlargement of a lump in the neck or between the ribs. Frequently there will be an increase in the size of the lung hernia with Valsalva manoeuvre. The hernial orifice is said to be easy to palpate and one can reduce the hernia by covering the orifice with the fingers. ${ }^{6}$ Diagnosis is usually evident clinically due to the change in size of the spongy mass with manoeuvres that change intrathoracic pressure, as in the case of our patient. Confirmation of the diagnosis can be accomplished with plain chest radiography utilising a tangential projection. Limited CT of the chest with Valsalva manoeuvre also usually demonstrates the hernia, but should be reserved for patients in whom the clinical or plain radiographic features are not conclusive.

Extrathoracic herniation of lung tissue is usually non-life-threatening and does not typically require major therapeutic interventional procedures. Out-patient management schemes include strapping the hernia site with compressive pads or by using a thoracic corset. Surgery should be considered when the hernia is causing chronic chest pain, ${ }^{9} 10$ incarcerates the pulmonary parenchyma, ${ }^{11} 12$ results in recurrent infection, or for cosmetic purposes. The use of local tissues and/or synthetic materials to repair the chest wall defect have been described. ${ }^{3} 91011$ 


\section{Final diagnosis}

Spontaneous intercostal herniation of a lung bulla in a patient with severe emphysema and cavitary lung disease.

1 Beale EC. On a case of hernia of the lung through diaphragma. Lancet 1882;1:139.

2 Munnell E. Herniation of the lung. Ann Thorac Surg 1968;5:204-12.

3 Scullion DA, Negus R, Al-Kutoubi A. Case report: extrathoracic herniation of the lung with a review of the literature. $\mathrm{Br} \mathcal{F}$ Radiol 1994;67:94-6.

4 Hiscoe DB, Digman GJ. Types and incidence of lung hernias. $\mathcal{F}$ Thorac Surg 1955;30:335-42.

5 Sloth-Nielsen J, Jurik AG. Spontaneous intercostal pulmonary hernia with subsegmental incarceration. Eur $\mathcal{F}$ Cardiothorac Surg 1989;3:562-4.

6 Montgomery JG, Lutz H. Hernia of the lung. Ann Surg 1925;82:220-31.

7 Prasad R, Mukerii PK, Gupta H. Herniation of the lung. Indian $\mathcal{F}$ Chest Dis Allied Sci 1990;32:129-32.
Keywords: lung bulla; herniation; mycobacterium; cavitary lung disease; bullous emphysema

8 Victor S, Muthurajan S, Sekhar TG, et al. Giant cervical herniation of an apical pumonary bulla. $\mathcal{F}$ Thorac Cardiovasc Surg 1987;93:141-7.

9 DiMarco AF, Oca O, Renston JP. Lung herniation: a cause of chronic chest pain following thoracotomy. Chest 1995; 107:877-9.

10 Cernilia J, Lin J, Ott R, Scannell G, Waxman K. A technique for repair of traumatic parasternal lung herniation. $\mathcal{F}$ Trauma 1995;38:935-6.

11 May AK, Chan B, Daniel TM, Young JS. Anterior lung herniation: another aspect of the seatbelt syndrome. $\mathcal{F}$ Trauma 1995;38:587-9.

12 Moncada R, Vade A, Gimenez C, et al. Congenital and acquired lung hernias. $\mathcal{F}$ Thorac Imaging 1996;11:75-82.

\title{
Mycobacterial liver abscess in a patient with AIDS
}

\author{
J L Casado, V Pintado, E Gomez-Mampaso, V Muñoz, M J Perez-Elías
}

A 33-year-old HIV-positive man was admitted to our hospital with a one-month history of lowgrade fever, malaise and weight loss. Eight years before he had been diagnosed with an asymptomatic liver hydatid cyst, which had not been treated. Two months before admission his CD4 count was 15 cells $/ \mu \mathrm{l}$. Physical examination showed a cachectic patient. Blood cultures taken on the first day of admission were negative, but a sputum smear revealed 10 acid-fast bacilli/100 fields on Ziehl-Neelsen staining. Abdominal ultrasonography revealed one large hypo-echoic lesion in the liver (figure 1), a finding confirmed by computed tomography (CT) (figure 2). Treatment with isoniazid, rifampin, ethambutol and pyrazinamide was started, but the patient remained febrile and showed progressive deterioration.

Infectious Diseases

Unit and Department of Microbiology, Hospital Ramón y Cajal, Madrid, Spain JL Casado

V Pintado

E Gomez-Mampaso

V Muñoz

MJ Perez-Elías

Correspondence to $\mathrm{JL}$ Casado, Infectious Diseases Unit, Hospital Ramón y Cajal, Cra Colmenar, km 9.1, 28034 Madrid, Spain

Accepted 20 August 1997

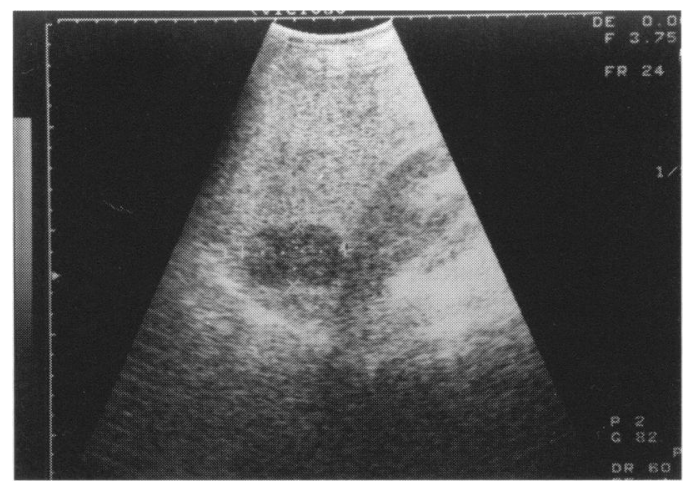

Figure 1 Abdominal ultrasound

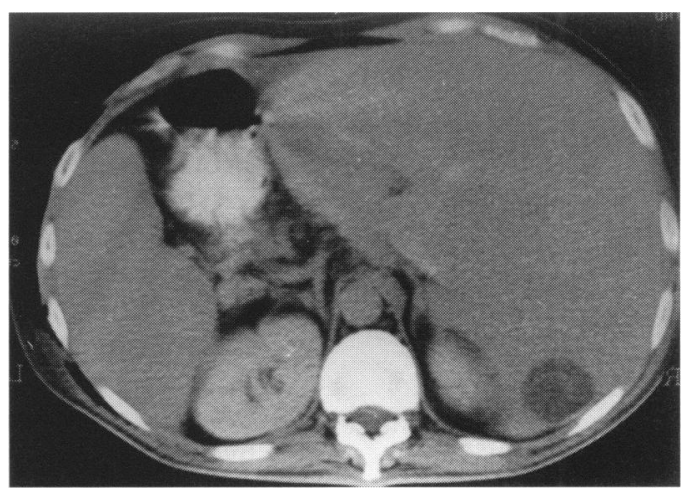

Figure 2 Abdominal CT scan

\section{Questions}

1 Which aetiologic agents should be considered?

2 How should the diagnosis be established?

3 What would be the best therapeutic approach ? 